\title{
Barriers to the early detection and intervention of children with autism spectrum disorders: A literature review
}

\author{
Fatema Ali Bivarchi ${ }^{1}$, Vahe Kehyayan*1, Sadriya Mohd Al-Kohji ${ }^{2}$ \\ ${ }^{1}$ University of Calgary in Qatar, Doha, Qatar \\ ${ }^{2}$ Primary Health Care Corporation, Doha, Qatar
}

Received: March 22, 2021

DOI: $10.5430 /$ jnep.v11n11p72
Accepted: July 7, 2021

Online Published: July 25, 2021

URL: https://doi.org/10.5430/jnep.v11n11p72

\begin{abstract}
Background and objective: Autism spectrum disorder (ASD) is a lifelong developmental disability that affects how individuals communicate and interact with others. A reliable diagnosis of ASD can be made within the first 24 months of a child's life, but ASD is usually diagnosed late. Late diagnosis contributes to missed opportunities to provide early intervention services and improve long-term outcomes. The purpose of this project was to identify barriers to early detection and intervention of ASD faced by parents, other caregivers, and health care professionals.

Methods: A literature review was conducted. CINAHL, Medline, and PsychINFO databases were used to search for relevant articles. Ten articles that met the inclusion criteria were selected and data from these articles were summarized in a data extraction table and themes were identified.

Results: Five main barriers that prevent early diagnosis and intervention of children with ASD were identified. These barriers were lack of knowledge, social stigma, dismissal of parents' first concerns by healthcare providers, barriers to ASD screening, and access to ASD services.

Conclusions: The results of this literature review will inform the development of an educational guide for parents and other caregivers to promote their knowledge and awareness about ASD in children.
\end{abstract}

Key Words: Autism, Autism spectrum disorder, Early detection, Early identification, Early intervention, Child, Toddler, Barrier, Caregiver, and parent

\section{INTRODUCTION}

Autism spectrum disorder (ASD) is a neurological and developmental disorder that causes an impairment in social interactions and poor communications with others as well as a set of challenging behaviors. ${ }^{[1]}$ These behavioral problems interfere with daily life skills and affect the thinking, learning, and problem-solving capabilities of children with ASD ${ }^{[2]}$ which can range from mild to severe. ${ }^{[3]}$ These be- haviors usually appear in early childhood and are inherent in these individuals throughout their life. ${ }^{[4]}$

The onset of ASD begins in the first two years of children's life. ${ }^{[4]}$ The American Academy of Pediatrics recommends a comprehensive screening for ASD at 18 and 24 months during well-child visits. ${ }^{[5]}$ There is evidence from biomedical and clinical research that early detection can increase opportunities to improve the developmental processes of children

*Correspondence: Vahe Kehyayan; Email: vkehyaya@ucalgary.ca; Address: University of Calgary in Qatar, Doha, Qatar. 
with $\mathrm{ASD},{ }^{[6]}$ and will lead to better prognosis and greater benefit. ${ }^{[7]}$ Early detection is also necessary to help reduce difficult behaviors, improve long-term outcomes, reduce the need for more expensive and costly interventions later in life, and increase the quality of life of children with ASD and their families. ${ }^{[2,4]}$

The subject of ASD in children and barriers to its early detection and intervention is of particular concern in the State of Qatar because of its increasing prevalence. A prevalence study of ASD in Qatar from 2015 to 2019 estimated a prevalence of $1.14 \%$ among children between the ages of 6 to 11 years old. ${ }^{[8]}$ As most children who suffer from ASD develop like other normal children, such as sitting, crawling, and walking on time in the first year of their life, ${ }^{[9]}$ some parents may not be aware about delays in other important developmental skills such as speech, social interaction, and communication skills. ${ }^{[10,11]}$ In consequence, they do not seek early intervention from their formal healthcare system. This is also true in the State of Qatar because of parents' lack of awareness of the early signs of ASD (personal communication, SA, 2020). Because of the importance to screen all children for ASD under the age of three, ${ }^{[12,13]}$ it is necessary to understand the reasons for delays in the early diagnosis and intervention for better health outcomes for children with ASD. Therefore, the purpose of this paper was to identify barriers to the early detection and intervention for ASD that prevent parents and other caregivers from accessing health care services and early interventions. Information about these barriers would be conducive to raise awareness in parents and healthcare professionals in Qatar, an Arabic and Muslim country in the Middle East, about potential barriers and the importance of early diagnosis and intervention in children with ASD.

\section{BACKGROUND}

Autism spectrum disorder is a neurodevelopmental disability. This disability in the central nervous system negatively affects the mental functions of the child, which leads to a decrease in cognitive performance and adaptive behavior in the child. ${ }^{[14]}$ The effects of ASD may vary from person to person according to the type and severity of symptoms of ASD. ${ }^{[15]}$ These symptoms range from mild to severe. ${ }^{[16]}$ Autism spectrum disorder occurs in all ethnic, racial, and socioeconomic groups ${ }^{[9,16]}$ It is characterized by difficulty in communication and building relationships with others (e.g., language expression, eye contact, and social interaction), as well as repetitive patterns of behaviors (e.g., repetitive hand and body movements; APA, 2013). These difficulties are present from early childhood and are inherent to individual with ASD throughout their lives. ${ }^{[2,6]}$

Published by Sciedu Press

\subsection{Importance of early detection and intervention}

Early detection and intervention can make a big difference in the lives of children with ASD. ${ }^{[17]}$ To provide ongoing care to these children and their families, pediatricians recommend that screening, diagnosis, and referral should be done as soon as possible. ${ }^{[18]}$ Research has shown that early detection of ASD is necessary to ensure that children have access to evidence-based interventions that aim to improve their developmental performance and obtain positive results in the future, such as improvement in language and social interactions. ${ }^{[6,9]}$ The early detection of ASD allows symptoms to be treated early when they appear, often between six to 18 months, rather than when the disorder fully appears. ${ }^{[19]}$ There is further substantial evidence from biomedical and clinical research that providing early childhood support can lead to improvements in the developmental processes and quality of life of children with ASD at all stages of their lives. ${ }^{[17]}$ The rationale for this is that children's brains are constantly growing and have a great capacity for formation and neuroplasticity at younger ages. ${ }^{[2]}$ Developing the skills and behaviors of children with ASD at an early age makes them less dependent on their caregivers and health care providers. ${ }^{[9]}$ Moreover, the availability and effectiveness of early intervention benefits children with ASD because they have to exercise their human rights to integrate into society, education, and play. ${ }^{[20]}$ Early detection is also an effective way to reduce the burden on and costs to parents and the community in the long term. ${ }^{[9,13]}$ Parents need to be aware of the importance of early recognition and diagnosis of ASD. Early diagnosis helps parents to establish and organize simple intervention plans. These plans help to decrease stress on parents, as they will be able to discuss and share their burden with the specialist physician and find an appropriate diagnosis for their children. ${ }^{[10]}$

\section{Methodology}

For the purpose of this study, a literature review was chosen. With the assistance of a librarian, a search strategy was developed. The search of the literature was conducted in the following databases: Cumulative Index of Nursing and Allied Health Literature (CINAHL), PsychINFO, and MEDLINE. The keywords searched were autism, "autism spectrum disorder", early detection, early identification, early intervention, child*, toddler*, barrier*, caregiver* and "parent*". The Boolean operators AND and OR were used to make this search more precise. An asterisk was used with some keywords to allow for a broader range of results. The following limiters were used to narrow the search result: peer-reviewed articles published between 2010 and 2019 and written in English. 


\subsection{Inclusion and exclusion criteria}

The inclusion criteria were qualitative and quantitative primary research studies that discussed ASD in children, explored barriers to early detection and intervention of children with ASD, and discussed implications to parents and caregivers. The exclusion criteria were articles that discussed etiology of autism, discussed ASD in adults, and included other special needs in children as well as articles that did not discuss barriers to early detection and intervention of children with ASD.

\subsection{Screening and evaluation of retrieved articles}

The initial search resulted in 393 articles. After removing duplicates, 370 articles remained. With the application of the limiters 117 articles were identified for possible inclusion. Screening of the titles and abstracts eliminated a further 67 articles. The remaining 50 articles were subjected to full-text review. Finally, based on the inclusion and exclusion criteria, a further 40 articles were eliminated leaving 10 articles for inclusion in this study. Appendix "A" shows the screening process and results.

\subsection{Methodological quality appraisal of articles}

The methodological quality of the 10 remaining articles was critically appraised independently by two of the authors (FAB and VK) using the Mixed Method Appraisal Tool (MMAT) version 2018. ${ }^{[21]}$ MMAT is designed to assess the quality of both quantitative and qualitative studies and mixed methods studies. It consists of two parts. The first part is a checklist of criteria that applies to all types of studies. The second part includes criteria applicable only to specific types of studies and provides explanations about the methodological quality criteria of these studies.

The critical appraisal determined that all 10 articles met the MMAT criteria in both its first and second parts. Of these 10 articles, five were qualitative studies (5 in USA and 1 each in Costa Rica, India, and Kenya) and two were quantitative ( 1 each in USA and India).

The 10 articles were organized in a summary table that includes the following categories: author, title, country, and year of publication; study design; sample and setting; and main findings (see Appendix "B"). This summary table assisted in presenting the data about barriers to early detection and intervention of children with ASD and identifying overarching themes.

\section{Findings}

Thematic analysis of the findings of the 10 articles identified five overarching themes as shown in Table 1. These themes were: (a) lack of knowledge, (b) social stigma, (c) access to ASD diagnostic services, (d) dismissal of parents' first reported concerns by healthcare providers, and (e) healthcare provider challenges to ASD screening. Several sub-themes were also identified.

Table 1. Overarching themes and sub-themes of barriers to the early detection and intervention of children with ASD

\begin{tabular}{|c|c|}
\hline Themes & Sub-themes \\
\hline \multicolumn{2}{|l|}{ Parents } \\
\hline - Lack of knowledge and education & $\begin{array}{l}\text { - Parents’ knowledge } \\
\text { - Education level of parents } \\
\text { - Healthcare care professionals' knowledge }\end{array}$ \\
\hline - Social stigma & - Isolation and rejection \\
\hline - Access to ASD diagnostic services & $\begin{array}{l}\text { - Lack of a clear path and information resources to access ASD services } \\
\text { - Financial burden }\end{array}$ \\
\hline \multicolumn{2}{|l|}{ Healthcare Providers } \\
\hline \multicolumn{2}{|c|}{ - Dismissal of parents' first reported concerns } \\
\hline - Challenges to ASD Screening & $\begin{array}{l}\text { - Inadequate time for physicians to screen ASD } \\
\text { - Lack of trained physicians and other healthcare providers to perform } \\
\text { ASD screening }\end{array}$ \\
\hline
\end{tabular}

\subsection{Barriers related to parents}

\subsubsection{Lack of knowledge}

Articles included in this literature review emphasized that a lack of knowledge and awareness among parents, caregivers, and professionals about ASD and its initial symptoms was a major barrier to early detection and intervention. The three sub-themes that derived from this theme were parents' knowledge, education level of parents, and health care professionals' knowledge.

The lack of knowledge among parents toward ASD and its early signs is a huge barrier to early detection and intervention of ASD. A qualitative study by Zuckerman et al. (2014) found that almost all parents in their study had a lack of 
knowledge about ASD before their children's diagnosis. ${ }^{[22]}$ In every focus group and interview of this study, parents stated "lack of knowledge" or "ignorance" about ASD as a problem in their Latino community. These authors found that parents did not understand what autism was, but they could describe symptoms of ASD based on the behaviors of their children. Similarly, a qualitative study by Schelly et al. (2019) included 54 parents to identify barriers that affected their information about an ASD diagnosis. ${ }^{[23]}$ Parents reported that they lacked knowledge about early symptoms of ASD. Nearly half of the parents knew nothing about ASD before their children were diagnosed. While some parents knew very little about ASD or had only heard about ASD from a movie, only three parents knew about specific symptoms of the disorder.

There is inconsistent evidence about parents' educational levels influencing their perception regarding early detection of ASD. For instance, Mahapatra et al. (2019) found that there was no correlation between the education level of parents and their lack of knowledge about ASD. ${ }^{[24]}$ These authors reported that although parents of children with ASD were well-educated and highly qualified, they still had poor knowledge and awareness about ASD. Similar findings were reported in the qualitative study by Elder et al. (2016). They reported that most of their participants still had poor understanding about ASD despite their high education and qualifications. ${ }^{[25]}$ Furthermore, Dababnah et al. (2018) found that mothers with college degrees or higher were afraid of labelling their children with ASD. ${ }^{[26]}$ As well, Khowaja et al. (2015) reported that there was a statistically significant correlation between parents' educational level and delayed diagnosis of ASD. ${ }^{[27]}$ In their study, mothers with a lower educational level were less likely to continue follow-up on ASD screening. They usually did not attend scheduled appointments, nor responded to scheduled calls; but mothers with the highest educational level showed the highest participation rate in ASD screening.

\subsubsection{Social stigma}

Social stigma is another barrier that contributes to delayed early diagnosis of ASD. Social stigma results in isolation and rejection. Kamau (2017) found that many parents, mostly mothers, faced a lot of challenges and suffered alone to raise their children with ASD. Parents reported how their children with ASD had been shunned by peers and other community members.

Although parents noticed some abnormal behaviors with their children, they ignored and did not accept that their children had these behaviors. ${ }^{[24]}$ Similar results were reported by Stahmer et al. (2019) who examined parents' and care- givers' perspectives on barriers to early diagnosis of ASD. ${ }^{[28]}$ They found that stigma related to ASD led to isolation and delayed acceptance of the diagnosis. Parents in their study reported that family and community members did not believe that their children had ASD and they ignored their concerns, which led to their reluctance to share the diagnosis of ASD. Parents also reported that community members attributed behavioral symptoms of ASD to a need for discipline and boys being boys, rather than a developmental concern. As well, these parents were blamed for poor parenting. Consequently, parents struggled on their own without family support.

Another major issue in families with children with ASD is most fathers leave after finding their child had ASD leading to the disintegration of families. ${ }^{[29]}$ Similar findings were also supported by Zuckerman et al. (2014) who found that parents believed that ASD was an embarrassing issue. Parents in their study initially denied that their children had ASD. They reported that they felt uncomfortable to share and discuss their children's developmental concerns with other people in the community and health care providers. Some mothers felt that fathers saw the existence of disabled boys as a weak reflection of them as men. As a result, fathers might not have believed in their wives' concerns, not cooperated with the diagnostic process, or might not have played a positive role in sharing the care of their children. ${ }^{[22]}$

\subsubsection{Access to ASD diagnostic services}

The lack of a clear pathway to access ASD services prevents parents from getting timely diagnosis and acquiring appropriate intervention services for their children with ASD. Elder et al. (2016) found that many parents struggled to access services and obtain a final diagnosis for their children. Parents reported that they faced challenges to navigate services from the first moment they suspected that something was not quite right with their children's development until the formal ASD diagnosis. ${ }^{[25]}$ Ribeiro et al. (2017) found that parents struggled to obtain their child's initial diagnosis of ASD because the diagnostic process was very long and complicated. ${ }^{[30]}$ For example, they spent 3 to 4 years to get a final diagnosis of ASD for their children. Access to services was also found to be limited by a lack of information and assistance. Parents reported that they had difficulties to obtain any kind of answers and any kind of assistance from health care providers of their children. ${ }^{[22]}$ These results were supported by Stahmer et al. (2019) who found that parents did not get the needed help and adequate information to navigate between ASD services and special education services for their children. ${ }^{[28]}$ There were also concerns that requirements for obtaining services were not explained in a coherent or family-friendly manner. Information as well was not disseminated in a systematic way and thus parents did not have sufficient awareness of the 
resources and services available. Zuckerman et al. (2014) reported that the lack of awareness campaigns about ASD in the media was another factor contributing to the limited availability of information among parents. Some parents reported that they never heard about ASD in the media. These parents emphasized the need for more educational outreach and attention to ASD in the media. ${ }^{[22]}$

Another contributing factor for limited access to services was due to lack of ASD specialists. Parents reported that lack of ASD professionals, especially in rural areas, made the diagnosis and intervention process difficult for them. ${ }^{[29]}$ Low availability of community resources prevented parents to obtain early routine screening for their children with ASD. ${ }^{[31]}$ Mahapatra et al. (2019) found that some parents had to travel to another city in search of a clear and informative pathway that could give a satisfactory diagnosis and intervention. Similar results were reported by Stahmer et al. (2019) that parents living in rural communities needed to travel long distances to find professionals who could guide and provide them with a definitive diagnosis and find the proper treatment for their children. ${ }^{[28]}$ Similarly, Elder et al. (2016) found that children of families living in rural and low-income areas were more likely to be delayed in ASD diagnosis.

Financial burdens in low-income families were another issue preventing them from accessing ASD services. ${ }^{[24]}$ Financial hardhips resulted in disconnection of telephones or families changing their phones frequently. ${ }^{[28]}$ Lack of transportation was another barrier to attending scheduling appointment. ${ }^{\text {[27] }}$ Other parents reported that lack of government or private insurance to cover the costs of autism treatment had put any chance of ASD treatment out of reach for most children. ${ }^{\text {[29] }}$ Parents reported that their low incomes prevented them from having good insurance coverage that would have helped their children to receive appropriate services. ${ }^{[25]}$

Mahapatra et al. (2019) found that costs associated with treatment and regular traveling led to a significant financial burden especially in low income families living in rural countries. ${ }^{[24]}$ Parents reported that most centers providing counseling and treatment for ASD were private centers, where they were required to pay a large sum of money for the treatment of their children. ${ }^{[24]}$ The complexities that parents faced to navigate and access ASD services consumed parents' time and delayed their childrens' diagnostic process. ${ }^{[22,24,25]}$

\subsection{Healthcare provider related barriers}

\subsubsection{Lack of knowledge, training}

Many primary care physicians as well lack knowledge and awareness of developmental milestones and how to assess them. Elder et al. (2016) reported that many physicians did not understand ASD and as a result did not really know what to look for. ${ }^{[25]}$ Because of their poor knowledge, they hesitated in providing early diagnosis. Instead, they just let the parents wait a long time without diagnosing their children or referring them to specialists at a young age. Other healthcare providers had misconceptions about ASD with most of them believing that ASD had a spiritual cause or it was a curse. ${ }^{[29]}$ Fenikile et al. (2015) explored potential barriers to the adoption of recommended screening for autism by family physicians at 18 and 24-month in child-care visits. ${ }^{[31]}$ They reported that family physicians not only lacked knowledge about the early signs of ASD, but also the specific ages when autism-specific screening was recommended. Most of the family physicians did not know the right questions to ask and even did not know what to look for at the critical age of 18 months.

\subsubsection{Dismissal of parents' first reported concerns}

The lack of parental credibility about their first concerns of ASD by healthcare providers is another pervasive theme that emerged in several studies regarding barriers to early diagnosis. Ribeiro et al. (2017) evaluated the pathway between initial parental concerns about atypical child development and ASD diagnosis. ${ }^{[30]}$ They found a significant relationship between first parental concerns about developmental delays in their children and a late diagnosis by pediatricians. Mothers had reported to healthcare professionals their concerns such as lack of eye contact, delay in verbal speech, and children not responding to their name. The pediatricians had dismissed the mothers' first concerns about the atypical development of their children and avoided asking about specific symptoms related to ASD, which delayed early detection of ASD. As as result, children were formally diagnosed with ASD not until the age of five years old, which is a delay of three years. These findings were supported by Dababnah et al. (2018) who reported that during the well-baby check-ups physicians did not take parents' initial concerns seriously and did not provide immediate referrals for ASD screening services with the result that children were diagnosed with autism later at the ages of seven and eight. ${ }^{[26]}$ Similar findings were reported by Ribeiro et al. (2017) and Stahmer et al. (2017).

\subsubsection{Challenges to ASD screening}

The lack of ASD screening in well-child visits contributes to delaying early diagnosis and timely intervention. This review highlighted two main barriers to ASD screening. These barriers were lack of time for physicians to do ASD screening and lack of trained clinicians to perform autism screening. Studies indicated that lack of time was a significant barrier to implementing routine screening in well-child care visits. ${ }^{[25,31]}$ Fenikilé et al. (2015) found that family physicians 
generally did not support routine screening for ASD during well-child visits and they had limited time to examine each child in a well-child care clinic. This limited time prevented physicians from screening and at the same time gathering relevant information from parents. These researchers reported that physicians faced challenges in balancing parental concerns, recommended health guidelines outlined in the practice guidelines, and time limitations. ${ }^{[31]}$

In order to give proper diagnosis and intervention programs for children with ASD, screening is extremely important as it is the first step before diagnosis. However, studies in this literature review indicated that a lack of awareness regarding routine ASD screening and screening tools among physicians led to delay in early diagnosis and intervention programs in children with ASD. ${ }^{[25,31]}$ Fenikilé et al. (2015) found that most physicians lacked familiarity with autism screening tools and were not aware of any specific guidelines recommending routine autism screening. ${ }^{[31]}$ Elder et al. (2016) suggested that the lack of knowledge about autism screening tools among physicians was due to their general lack of knowledge about autism. ${ }^{[25]}$ Participants recognized the need to emphasize developmental issues among professionals during training and continuing professional education to improve good child-care practices.

\section{Discussion}

This literature review aimed to highlight potential barriers to the early detection of ASD and its timely intervention. The findings of this literature review may help parents, caregivers, and health care providers understand the factors that influence their knowledge about early detection and intervention of ASD. These findings will also provide some recommendations in enhancing the knowledge of patients, caregivers, and healthcare providers and improving their awareness about ASD in young children.

Five major themes emerged from the thematic analysis regarding barriers to early detection and intervention from this literature review. These themes were lack of knowledge, social stigma, health-care providers' rejection of parenting first concerns, barriers to screening ASD, and access to ASD diagnostic services. Eight subthemes were also identified.

This literature review emphasized that parents lacked knowledge and awareness about early signs of ASD, which prevented them from understanding developmental delays leading to delayed early diagnosis and intervention. ${ }^{[22,23,25,29]}$ This finding is consistent with Chu et al.'s (2018) study that found that the majority of parents lacked knowledge about early signs and symptoms of ASD and how to manage their children's unique behaviours, such as refusing to socialize

Published by Sciedu Press with their peers, limited eye contact, and speech-language delays. All parents had reported that this was their first child with ASD and that they had no previous experience with it. They had become aware of ASD only after their child had been diagnosed by a specialist. ${ }^{[11]}$ The findings of this literature review also showed that parents' knowledge about ASD was influenced by their level of education. ${ }^{[24-26]}$ This finding was supported by Anwar et al.'s (2018) study that indicated that parents with higher education level showed more knowledge about early signs of ASD and were able to manage the behaviours of their children more than parents with lower education levels. However, a study by Deeb (2016) indicated that there were no significant differences between parents' level of education and their knowledge about managing their children's behaviours. ${ }^{[32]}$

Lack of knowledge about the early signs of ASA and how to assess them was also common among healthcare providers. Consequently, children with ASD were not referred to specialists for early diagnosis. ${ }^{[22-25,27,29]}$ This finding was supported by Al-Farsi et al. (2016) who found that physicians lacked knowledge about ASD and were not equipped to fully recognize early signs of ASD in children. ${ }^{[33]}$ As well, Walsh et al. (2020) showed that the lack of trained health care providers to provide parents with adequate information and guide them in obtaining information about an appropriate intervention made the process of obtaining ASD services more difficult. ${ }^{[34]}$

Another barrier that prevents caregivers from the early detection of ASD in their children is social stigma. Social stigma related to ASD leads to isolation and delayed acceptance of the diagnosis among family and community members. ${ }^{[22,26,28,29]}$ This finding is supported by findings of Guler et al. (2018). ${ }^{[35]}$ Their study showed that community members blamed parents for their children's behaviors. Many parents reported they were feeling lonely because their friends and family did not understand what they were experiencing. Friends and family members thought that their children were "naughty," or the parents did not teach their children proper manners. ${ }^{[35]}$ Similarly, a study by Gona et al. (2016) found that caregivers of children with ASD expressed feelings of isolation and shame when others mislabeled their children as naughty. ${ }^{[36]}$ Most parents in their study reported that people around them in the community consistently blamed them for raising their children in a bad way, and they were seen as irresponsible parents. Many parents reported that children with ASD are rejected by peers, family members, relatives, and the entire community. Lack of family and community support for caregivers resulted in isolation and delays in accepting their child's diagnosis. Therefore, these parents need social support to overcome 
these difficulties. ${ }^{[36]}$

This review found that difficulties in navigating and accessing ASD services as well as the financial burden faced by caregivers were associated with delays in the diagnostic process of ASD. ${ }^{[22,24,25,27-31]}$ This finding is consistent with Chao et al.'s (2018) findings that many parents struggle to obtain ASD services for their children. Most parents had reported that they received various information about ASD services from their healthcare providers, which made seeking out ASD services more confusing and complicated. ${ }^{[15]}$ Neupane (2020) suggested that parents needed to be guided to existing ASD services early at the time of diagnosis. ${ }^{[37]}$

Zarafshan et al. (2019) also showed that the lack of accessible ASD services prompted parents to travel to another region to get more intervention services for their children. Parents spend much time and go long distances to access the services. ${ }^{[18]}$ Financial burden was another factor making accessing ASD services more difficult.

Low-income families use almost all of their money for the treatment of their children with ASD. ${ }^{[38]}$ The lack of insurance coverage, the need to take time off work to look after their children, and the lack of transportation to take their children to appointments all place a huge financial burden on parents. [38]

For the early detection of ASD, it is very important for healthcare providers to pay attention to parents' reported concerns about the developmental issues of their children. This review highlighted that healthcare providers ignore parents' initial concerns about ASD, resulting in delayed early diagnosis of ASD for their children. ${ }^{[22,25,28,30]}$ This finding is in line with Neupane's (2020) study that found that parents were dissatisfied with their primary caregivers. Parents reported that health care providers ignored their concerns and told them to "wait and see" without taking any action to deal with the parents' concerns. Parents blame healthcare providers for this delay. ${ }^{[37]}$ A study by Zuckerman et al. (2015) found also that most parents who did not receive attention about their early developmental concerns from healthcare providers were more likely to have a delay in the diagnosis of their children with a delay of nearly three years after the first consultation they had with the healthcare provider. ${ }^{[39]}$ Similarly, Zarafshan et al. (2019) showed that the most common concerns parents reported about their children to primary health care providers were lack of communication and delayed speech. These parents reported that primary care providers did not pay attention to their concerns and, instead, reassured them that there was nothing to worry about. ${ }^{[18]}$ A study by Zuckerman et al.'s (2015) found that parents who received more proactive responses by healthcare providers to their children's developmental concerns had shorter delays in diagnosing ASD.

Finally, this review also showed that the lack of clinicians' time to do ASD screening and training to perform ASD screening were also major barriers to the delayed detection and diagnosis of ASD in children. ${ }^{[25,31]}$ This finding is in line with Yerramsetti's (2017) study that showed that healthcare providers and professionals did not have sufficient time to perform routine screening for each child during regular screening and that they had difficulties managing children with ASD. ${ }^{[40]}$ While pediatricians recognized the potential benefits of ASD screening, they also pointed out that they faced many challenges in implementing routine screening. ${ }^{[41]}$ These challenges included lack of time, lack of familiarity with the use of screening tools, and difficulty in balancing development monitoring with formal examination. Therefore, pediatricians preferred to refer children to a developmental specialist rather than using any screening measures once they learned of the parents' concerns. ${ }^{[41]}$ Moreover, Zarafshan et al. (2019) reported that the lack of ASD screen training among health care providers had limited their role in managing and caring for children with ASD. These authors suggested that health care providers needed to be well-trained to be familiar with using screening tools to diagnose ASD based on standard protocols and tests. They suggested the appointment of expert physicians to assist in the assessment of these children in order reduce such difficulties. The increase in the number of expert physicians may help to facilitate the development of screening procedures that are consistent in busy practices. ${ }^{[18]}$

In sum, a thematic analysis of the peer-reviewed literature identified that early detection of ASD in children is influenced by the knowledge of parents, caregivers, and healthcare providers, social stigma, lack of attention by healthcare professionals to parents' concerns, barriers to ASD screening, and access to ASD services.

\subsection{Strengths and limitations}

This literature review has several strengths. First, a systematic search method was used to find quality articles. A second strength was that the included articles were primary studies including quantitative and qualitative studies. Another strength was that barriers to the early diagnosis and intervention of ASA in children were common among caregivers and health care providers in different communities and different contexts around the world, such as Brazil, Costa Rica, India, Kenya, and the United States of America. While no such studies have been conducted in Qatar to the best knowledge of the authors, the identified barriers might be applicable to Qatar's context. 


\subsection{Implications and recommendations}

This literature review identified many barriers that influenced early detection and intervention among children with ASD. It is important to address these barriers that prevent parents from the early diagnosis and treatment of their children, such as their lack of knowledge about ASD and its early signs. As parents are the primary caregivers of their children and they are best positioned to recognize developmental issues in their children, an evidence-based educational guide tailored to parents is recommended. It is also important to expand the scope of primary care services to improve early detection and intervention as well as expand access to screening and diagnostic facilities in the community. Education of parents, caregivers and healthcare providers would have the potential to achieve better health outcomes for children with ASD.

\section{Conclusion}

The early detection and intervention of ASD play an important role in improving developmental and behavioral out- comes in children with ASD. The aim of this literature review was to identify potential barriers to early detection and intervention of ASD faced by parents, other caregivers, and healthcare professionals. The main barriers that have been reported in this literature review were lack of knowledge, social stigma, lack of access to ASD services, dismissal of parents' first reported concerns by health care providers, and challenges to ASD screening. The findings of this literature review may help parents and other caregivers to better manage their children and allow healthcare providers to identify the gaps in their clinical practice of early routine screening and management of children with ASD. Based on the findings of this literature review, education of parents, other caregivers and healthcare providers is recommended to improve their knowledge and skills in the early identification, assessment, and intervention of ASD in children.

\section{CONFLicts OF INTEREST Disclosure}

The authors declare no conflict of interest.

\section{REFERENCES}

[1] American Psychiatric Association. Diagnostic and statistical manual of mental disorders. 5th ed. Washington, DC: American Psychiatric Association Publishing; 2013. https ://doi .org/10.1176/appi . books. 9780890425596

[2] Zwaigenbaum L, Brian JA, Ip A. Early detection for autism spectrum disorder in young children. Paediatrics \& Child Health. 2019; 24: 424-43. PMid:31660041 https://doi .org/10.1093/pch/pxz1 19

[3] Sanchack KE, Thomas CA. Autism Spectrum Disorder: Primary Care Principles. Am Fam Physician. 2016; 94: 972-9.

[4] Elder JH, Kreider CM, Brasher SN, et al. Clinical impact of early diagnosis of autism on the prognosis and parent-child relationships. Psychology Research \& Behavior Management. 2017; 10: 283-92. PMid:28883746 https : //doi .org/10.2147/PRBM.S117499

[5] Monteiro SA, Dempsey J, Berry LN, et al. Screening and Referral Practices for Autism Spectrum Disorder in Primary Pediatric Care. Pediatrics. 2019; 144: 10. PMid:31515298 https ://doi .org/10 .1542/peds . 2018-3326

[6] Hahler EM, Elsabbagh M. Autism: A global perspective. Current Developmental Disorders Reports. 2015; 2: 58-64. https: //doi.org/10.1007/s40474-014-0033-3

[7] Blumberg SJ, Zablotsky B, Avila RM, et al. Diagnosis lost: Differences between children who had and who currently have an autism spectrum disorder diagnosis. AUTISM. 2016; 20: 783-95. PMid:26489772 https://doi.org/10.1177/13623613156077 24

[8] Alshaban F, Aldosari M, Al-Shammari H, et al. Prevalence and correlates of autism spectrum disorder in Qatar: a national study. Journal of Child Psychology \& Psychiatry. 2019; 60: 1254-68. PMid:31069792 https://doi.org/10.1111/jcpp.13066

[9] Fein D, Barton M, Dumont-Mathieu T. Optimizing outcome in autism spectrum disorders. Policy Insights from the Behavioral and Brain
Sciences. 2017; 4: 71-8. https://doi.org/10.1177/23727322 16685098

[10] Anwar MS, Tahir M, Nusrat K, et al. Knowledge, Awareness, and Perceptions Regarding Autism Among Parents in Karachi, Pakistan. Cureus. 2018; 10: e3299.

[11] Chu SY, Mohd Normal SNSAB, McConnell GE, et al. Challenges faced by parents of children with autism spectrum disorder in Malaysia. Speech, Language and Hearing. 2018; 23: 221-31. https : //doi.org/10.1080/2050571X.2018.1548678

[12] Koegel LK, Koegel RL, Ashbaugh K, et al. The importance of early identification and intervention for children with or at risk for autism spectrum disorders. International Journal of Speech-Language Pathology. 2014; 16: 50-6. PMid:24328352 https : //doi .org/10.310 $9 / 17549507.2013 .861511$

[13] Zwaigenbaum L, Bauman ML, Choueiri R, et al. Early Intervention for Children With Autism Spectrum Disorder Under 3 Years of Age: Recommendations for Practice and Research. Pediatrics. 2015; 136 : S60-81. PMid:26430170 https ://doi.org/10.1542/peds. 201 4-3667E

[14] World Health Organization. Autism spectrum disorder. 2019.

[15] Kuo-Yu Chao, Hsueh-Ling Chang, Wei-Chih Chin, et al. How Taiwanese parents of children with autism spectrum disorder experience the process of obtaining a diagnosis: A descriptive phenomenological analysis. AUTISM. 2018; 22: 388-400. PMid:28205453 https://doi.org/10.1177/1362361316680915

[16] Baio J, Wiggins L, Christensen DL, et al. Prevalence of Autism Spectrum Disorder Among Children Aged 8 Years - Autism and Developmental Disabilities Monitoring Network, 11 Sites, United States, 2014. MMWR SURVEILLANCE SUMM. 2018; 67: 1-23. PMid:29701730 https ://doi.org/10.15585/mmwr.ss6706a1

[17] Lord C, Brugha TS, Charman T, et al. Autism spectrum disorder. Nature Reviews. 2020; 6(1): 5. PMid:31949163 https : //doi .or $\mathrm{g} / 10.1038 / \mathrm{s} 41572-019-0138-4$ 
[18] Zarafshan H, Mohammadi MR, Abolhassani F, et al. Current Status of Health and Social Services for Children with Autism in Iran: Parents' Perspectives. Iranian Journal of Psychiatry. 2019; 14: 76-83. https://doi.org/10.18502/ijps.v14i1.427

[19] Tanner A, Dounavi K. The Emergence of Autism Symptoms Prior to 18 Months of Age: A Systematic Literature Review. Journal of Autism \& Developmental Disorders. 2020 Jul 30.

[20] Barton EE, Harris B, Leech N. Autism Identification Policies and Practices in Early Childhood. INFANTS YOUNG CHILD. 2016; 29: 267-89. https://doi.org/10.1097/IYC.0000000000000076

[21] Hong QN, Pluye P, Fàbregues S, et al. Mixed Methods Appraisal Tool (MMAT), Version 2018. 2018.

[22] Zuckerman KE, Sinche B, Mejia A, et al. Latino parents' perspectives on barriers to autism diagnosis. Academic Pediatrics. 2014; 14: 301-8. PMid:24767783 https://doi.org/10.1016/j . acap .2013 .12 .004

[23] Schelly D, Gonzalez PJ, Solis PJ. Barriers to an Information Effect on Diagnostic Disparities of Autism Spectrum Disorder in Young Children. Health Services Research \& Managerial Epidemiology. 2019.

[24] Mahapatra P, Pati S, Sinha R, et al. Parental care-seeking pathway and challenges for autistic spectrum disorders children: A mixed method study from Bhubaneswar, Odisha. Indian Journal of Psychiatry. 2019; 61: 37-44. PMid:31896885 https ://doi.org/10.410 3/psychiatry. IndianJPsychiatry_291_19

[25] Elder JH, Brasher S, Alexander B. Identifying the Barriers to Early Diagnosis and Treatment in Underserved Individuals with Autism Spectrum Disorders (ASD) and Their Families: A Qualitative Study. Issues Ment Health Nurs. 2016; 37: 412-20. PMid:27070190 https://doi.org/10.3109/01612840.2016.1153174

[26] Dababnah S, Shaia WE, Campion K, et al. "We Had to Keep Pushing": Caregivers' Perspectives on Autism Screening and Referral Practices of Black Children in Primary Care. INTELLECT DEV DISABIL. 2018; 56: 321-36. PMid:30273522 https ://doi .org/ 10.1352/1934-9556-56.5.321

[27] Khowaja M, Hazzard A, Robins D. Sociodemographic Barriers to Early Detection of Autism: Screening and Evaluation Using the MCHAT, M-CHAT-R, and Follow-Up. J Autism Dev Disord. 2015; 45 1797-808. PMid:25488122 https://doi.org/10.1007/s10803 $-014-2339-8$

[28] Stahmer AC, Vejnoska S, Iadarola S, et al. Caregiver Voices: CrossCultural Input on Improving Access to Autism Services. Journal of Racial \& Ethnic Health Disparities. 2019; 6: 752-73. PMid:30859514 https://doi.org/10.1007/s40615-019-00575-y

[29] Kamau LZ. Autism spectrum disorders (ASD) in Kenya: Barriers encountered in diagnosis, treatment and management. J. Res. Pharm. Sci. 2017; 3: 1-11.
[30] Ribeiro SH, Paula CSd, Bordini D, et al. Barriers to early identification of autism in Brazil. Revista Brasileira de Psiquiatria. 2017; 39: 352-4. PMid:28977067 https ://doi .org/10.1590/1516-4 446-2016-2141

[31] Fenikilé TS, Ellerbeck K, Filippi MK, et al. Barriers to autism screening in family medicine practice: a qualitative study. PRIM HEALTH CARE RES DEV (CAMBRIDGE UNIVERSITY PRESS/UK). 2015; 16: 356-66. PMid:25367194 https://doi.org/10.1017/S146 3423614000449

[32] Deeb RMAS. Knowledge of parents of children with autism spectrum disorder of behavior modification methods and their training needs accordingly. International Education Studies. 2016; 9: 141-54. https://doi.org/10.5539/ies.v9n10p141

[33] Al-Farsi YM, Al Shafaee MA, Al-Lawati KS, et al. Awareness about autism among primary healthcare providers in Oman: A crosssectional study. Global Journal of Health Science. 2016; 9: 65-75. https://doi.org/10.5539/gjhs.v9n6p65

[34] Walsh C, Lydon S, O'Dowd E, et al. Barriers to Healthcare for Persons with Autism: A Systematic Review of the Literature and Development of A Taxonomy. DEV NEUROREHABIL. 2020; 23: 413-30. https://doi.org/10.1080/17518423.2020.1716868

[35] Guler J, de Vries PJ, Seris N, et al. The importance of context in early autism intervention: A qualitative South African study. AUTISM. 2018; 22: 1005-17. PMid:28914083 https://doi.org/10.1177/ 1362361317716604

[36] Gona JK, Newton CR, Rimba KK, et al. Challenges and coping strategies of parents of children with autism on the Kenyan coast. RURAL REMOTE HEALTH. 2016; 16: 1-12.

[37] Neupane KG. Autism Spectrum Disorder: The Parental Experience. J Psychosoc Nurs Ment Health Serv. 2020; 58: 14-9. PMid:31710368 https://doi.org/10.3928/02793695-20191022-02

[38] Martinez M, Thomas KC, Williams CS, et al. Family Experiences with the Diagnosis of Autism Spectrum Disorder: System Barriers and Facilitators of Efficient Diagnosis. Journal of Autism \& Developmental Disorders. 2018; 48: 2368-78. PMid:29453706 https://doi.org/10.1007/s10803-018-3493-1

[39] Zuckerman KE, Lindly OJ, Sinche BK. Parental concerns, provider response, and timeliness of autism spectrum disorder diagnosis. J Pediatr. 2015; 166: 1431,9.e1. PMid:25888348 https://doi.org/ $10.1016 / j \cdot j$ peds .2015 .03 .007

[40] Yerramsetti AP. 29.5 The Other Patients: Caregiver and Clinician Stress in Treating Autism Spectrum Disorder. Journal of the American Academy of Child \& Adolescent Psychiatry. 2017; 56: S45. https://doi.org/10.1016/j.jaac.2017.07.176

[41] Ip AWS, Zwaigenbaum L, Nicholas D, et al. Factors influencing autism spectrum disorder screening by community paediatricians. Paediatrics \& Child Health. 2015; 20: e20-4. PMid:26175565 https://doi.org/10.1093/pch/20.5.e20 